\title{
Experimental investigation of plasma instabilities by Fourier analysis in an electron cyclotron resonance ion source
}

\author{
Sarvesh Kumar, ${ }^{1, *}$ Jyotsna Sharma, ${ }^{2, \dagger}$ Prashant Sharma, ${ }^{1}$ Shatendra Sharma, ${ }^{3}$ \\ Yaduvansh Mathur, ${ }^{1}$ Devendra Sharma, ${ }^{4}$ and Manish K. Kashyap ${ }^{5}$ \\ ${ }^{1}$ Inter University Accelerator Centre, Aruna Asaf Ali Marg, New Delhi 110067, India \\ ${ }^{2}$ Amity School of Applied Sciences, Amity University, Gurgaon 122413, Haryana, India \\ ${ }^{3}$ USIC, Jawaharlal Nehru University, New Delhi 110067, India \\ ${ }^{4}$ Institute for Plasma Research, HBNI, Bhat, Gandhinagar 382428, Gujarat, India \\ ${ }^{5}$ Department of Physics, Kurukshetra University, Kurukshetra 136119, Haryana, India
}

(Received 12 March 2018; published 14 September 2018; corrected 16 October 2018)

\begin{abstract}
The plasma instabilities play an important role in an electron cyclotron resonance (ECR) ion source for the production of intense heavy ion beams in high charge states for particle accelerators. The geometrical and operational constraints of ECR sources hinder the trapping of ions for a sufficient time to get fully ionized with maximum efficiency. This problem is looked at in detail by studying the plasma instabilities in ECR ion sources. The ECR environment is full of complex rearrangements of various electric and magnetic fields to define a sustainable trap for the ions. The maximum frequency of plasma instability has been observed to be of $122.5 \mathrm{kHz}$ under a set of sustainable plasma parameters. However, this limit may be pushed further if the plasma is overdriven in terms of source parameters. The instabilities cover a full regime of few tens of $\mathrm{Hz}$ to few hundreds of $\mathrm{kHz}$ under various operating conditions of radio frequency (rf), negative bias voltage, rf power and injection gas pressure. The rigorous details of frequencies and amplitudes of plasma instabilities are being reported by studying the Fourier spectrum of extracted and analyzed beam intensity. The plasma instabilities are attributed as drift waves in an inhomogeneous ECR plasma generated by the application of radio-frequency fields.
\end{abstract}

DOI: 10.1103/PhysRevAccelBeams.21.093402

\section{INTRODUCTION}

Electron cyclotron resonance (ECR) ion sources [1,2] are one of the best sources to boost the performance as well as the efficiency of modern particle accelerators and colliders in different fields of physics, especially fundamental research on heavy ions such as nuclear and atomic physics, etc. It is an ongoing quest to develop an ion source which maximizes the ion charge state, beam current and minimizes the beam emittance. Being capable of producing high beam currents and high charges states of heavy ions, the ECR sources are the main injectors for heavy ion accelerators. The production of highly charged ions is strictly governed by the inherent and induced plasma instabilities in the ECR ion sources. They can kill or enhance a particular charge state fraction depending on the freedom of recombinations for the species on the ECR surface, a

\footnotetext{
*sarvesh@iuac.res.in; sariuac@gmail.com

jsharma@ggn.amity.edu
}

Published by the American Physical Society under the terms of the Creative Commons Attribution 4.0 International license. Further distribution of this work must maintain attribution to the author(s) and the published article's title, journal citation, and DOI. magnetic confinement region where the Larmor frequency of electrons is equal to injected radio-frequency waves. The energy of electrons is boosted maximum to few $\mathrm{keV}$ by ECR mechanism and these energetic electrons also known as hot electrons, produce ionization by successive impacts to heavy ions. It demands longer plasma confinement time, high ionization rates and significant population of hot electrons to get a good fraction of fully stripped heavy ion beams. Thus, there is a quest to boost the magnetic field confinement by superconducting magnets and accordingly radio frequency using highly efficient klystrons in modern ECR technology. Electron beam ion trap sources are also known for generating higher charge states nearly fully stripped for heavy ions but lack in beam currents. On the other hand, ECR sources are productive for higher beam currents of high charge states of heavy ions but not for fully stripped ions. Thus, for ECR sources, in order to obtain the maximum current of fully stripped ions, the plasma instabilities have to be investigated under the complete range and combinations of its tuning parameters.

Plasma instabilities are studied experimentally in Q-machines and theoretically by various groups worldwide in the last six decades but there are few reports in the literature to study them in detail as per the multiple tuning 
parameters of the ECR ion sources by means of Fourier analysis of beam intensity. Recently, Tarvainen et al. [3,4] reported an experimental observation of electron cyclotron instabilities in the minimum-B ECR plasma which led to $\mathrm{ms}$ scale oscillations in extracted beam currents. They monitored the microwave emission, bremsstrahlung radiation and extracted beam current oscillation which happen in the regime of $\mathrm{ns}, \mu \mathrm{s}$, and $\mathrm{ms}$, respectively. They observed a maximum beam current oscillation up to a few $\mathrm{kHz}$. The plasma instabilities are also realized in terms of drift waves and researchers have reported many studies $[5,6]$ on drift waves in different plasma environments. The drift waves have been found to exist in ECR plasmas which are initially demonstrated in the laboratory by Hendel et al. [7], who detected waves either by ion-density or plasma potential fluctuations with Langmuir probes and explained theoretically by Chu et al. [8]. Drift wave turbulence can also be considered as universal instabilities in any magnetically confined plasma with a density gradient due to diamagnetic drift. It is a dominant source of anomalous transport in tokamaks and stellarators. Semet et al. [9] observed the low-frequency microturbulence using far-infrared laser scattering measurements from a tokamak plasma. A drift wave model for inward energy transport in tokamak plasmas is given by Weiland et al. [10]. Gore et al. [11] reported experimentally the suppression of the entire drift wave spectrum by externally applied rf fields of few watts power in a Q machine. Gopalraja et al. [12] demonstrated also experimentally the suppression of drift waves by an externally launched lower hybrid wave in an ion beam plasma system and attributed to the ponderomotive force that enhances the Landau damping of electrons. Theoretically, Fainberg et al. [13] and Okamoto et al. [14] considered the application of a high frequency near electron plasma frequency in which the oscillating electric field acts along the direction of the magnetic field and found the possibility of stabilizing drift instabilities in an inhomogeneous plasma. It is shown that the collisionless drift instability can be stabilized by a longitudinal electric field under certain conditions. In a single-ended Q machine, Schrittwiser et al. [15] showed experimentally that drifting electrons without the formation of a localized twodimensional sheath are able to excite the ion-cyclotron instability. The frequency of instability (few $\mathrm{kHz}$ to hundreds of $\mathrm{kHz}$ ) is found to increase linearly with the magnetic field. These results were in agreement with the work of Drummond et al. [16] where ion acoustic waves and electrostatic ion cyclotron waves exist under certain conditions of thermal and drift velocities of both electron and ions. Sharma et al. [17] calculated the excitation of electrostatic ion-cyclotron waves by an ion beam in a twoion component plasma and found that the unstable wave frequencies of both the light and heavy positive ion modes increase with the magnetic fields in accordance with the experimental observations of Suszcynsky et al. [18].
They also calculated the excitation of lower hybrid waves [19] by a gyrating ion beam in a negative ion plasma and found the similar observation.

\section{EXPERIMENTAL DETAIL AND OBSERVATIONS}

In the present work, the plasma instabilities are studied in a low volume, $10 \mathrm{GHz}$ compact Nanogan ECR ion source $[20,21]$ which is placed on a $400 \mathrm{kV}$ high voltage platform. A schematic diagram of the source is shown in Fig. 1(a) along with an analyzing dipole magnet and beam diagnostics measurement system of a wire scanner type beam profile monitor (BPM) and a Faraday cup (FC). Radiofrequency power from a traveling wave tube amplifier ( 0 to $250 \mathrm{~W}, 8-18 \mathrm{GHz}$ ) is fed directly using a WR62 waveguide into the ECR plasma chamber with the dc break and a vacuum window in between for providing vacuum and high voltage isolation to the microwave amplifier section. Normally, here rf power $\leq 20 \mathrm{~W}$ is enough to generate a plasma to extract hundreds of microampere beam currents [22]. The power density is quite high due to the small volume of Nanogan ECR source and is somewhat comparable to the case of high volume and high rf power ECR sources. The signal from the Faraday cup is analyzed in the frequency domain using spectrum analyzers (SA) namely HP 3582A and Keysight N9000A-503 CXA working in low- and high-frequency domains, respectively. The errors estimated in tuning parameters of the source as well as in diagnostics measurements are $\leq 0.1 \%$. The beam currents are of the order of few microamperes for different charge states of the oxygen beam and get modulated as per the instability frequencies existing in the ECR plasma. It reflects as distinct peaks in the Fourier spectrum of beam intensity. The energies for various oxygen beams are

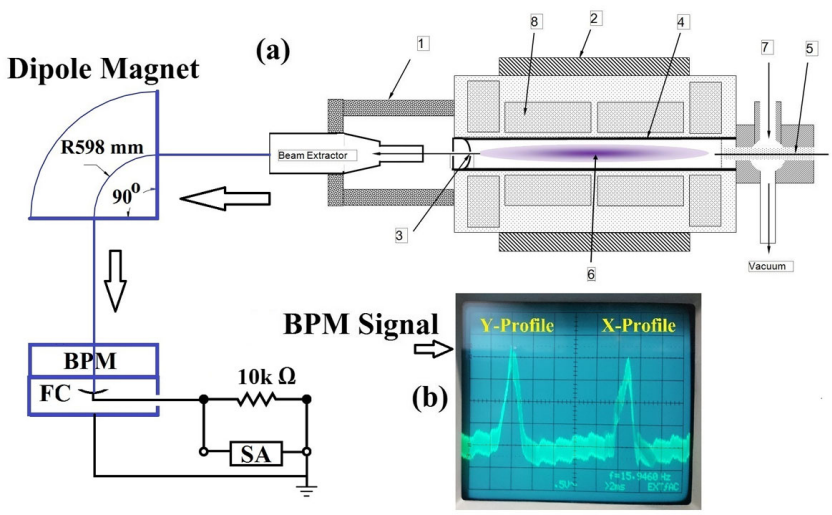

FIG. 1. (a) Schematics of ECR ion source with dipole magnet and beam diagnostics: 1-high voltage insulator; 2-source body; 3-plasma electrode; 4-plasma chamber; 5-negative dc bias; 6-ECR zone; 7-rf injection line; 8-permanent magnetic confinement both axial and radial. (b) Transverse beam profiles from BPM corresponding to maximum plasma instability amplitude. 
$q \times 250 \mathrm{keV}$ where $\mathrm{q}$ is the charge state of the analyzed oxygen beam.

The Nanogan ECR source [23-25] has an all permanent magnetic confinement using $\mathrm{NdFeB}$ structure where the electrons diffuse from the high-pressure injection side to the low-pressure extraction side within the confined plasma. In order to confine the plasma in all possible directions, the magnetic field due to a Halbach type multipole increases radially towards the chamber walls for radial confinement and a longitudinal field due to solenoids creates magnetic mirror configuration with loss cone at the extraction side of the ion source. Due to this confinement, a closed egg-shaped ECR surface is formed where the electrons lose or gain energy relative to their phase with respect to the injected radiowaves. The electrons, in turn, ionize the atoms and molecules of the particular species of interest to finally form a stable plasma. Larmor radius $\rho_{e}(\mathrm{~mm})$ of electrons with energy (E) in a confined field (B) is given as $0.035 \sqrt{E(\mathrm{eV})} / B(\mathrm{kG})$. In $\mathrm{ECR}$, the resonance field for the electrons at the operational frequency of $10 \mathrm{GHz}$ is $B_{\mathrm{ECR}}=3.571 \mathrm{kG}$. Hence, for a range of energies from $1 \mathrm{eV}$ to $1 \mathrm{keV}$, the Larmor radius varies from $9.8 \mu \mathrm{m}$ to $0.31 \mathrm{~mm}$ for cold to hot electrons. These electrons are capable of producing ionizations depending upon their kinetic energy and confinement time.

The source is capable of producing hundreds of microampere beam current but it is restricted to few microamperes at the Faraday cup which is enough and well within safe operational range of spectrum analyzer without attenuators. Thus, all the studies reported in this section are performed at low rf powers. The oxygen beam is tuned at $250 \mathrm{kV}$ potential from the ECR source. The beam is analyzed in the 90-degree beam line of a switching cum analyzing magnet [23] for possible charge states of oxygen ions. The mass spectrum is taken by scanning the magnetic field and observing the currents of the analyzed beam on the Faraday cup and is shown in Fig. 2. The plasma instability frequency is depicted by the Fourier spectrum of extracted and analyzed beam current oscillations. Its maximum value is found to be $122.5 \mathrm{kHz}$ with a significant

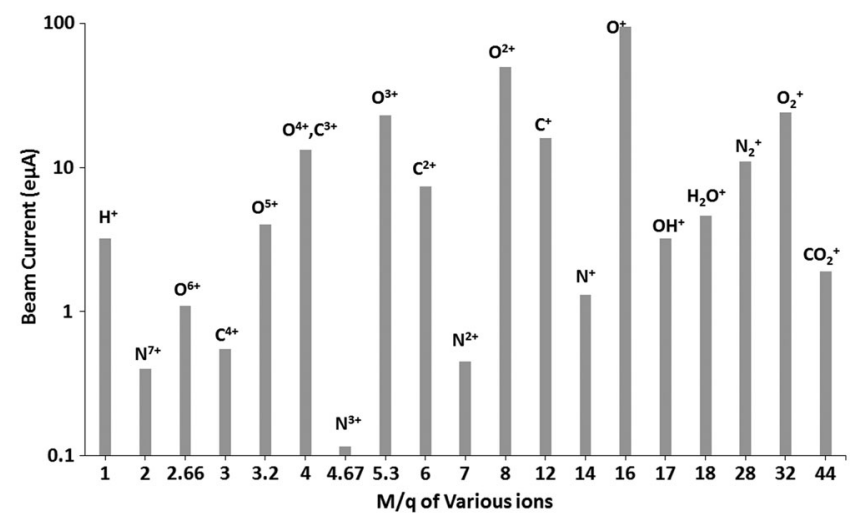

FIG. 2. Mass spectrum of analyzed beams from ECR source.

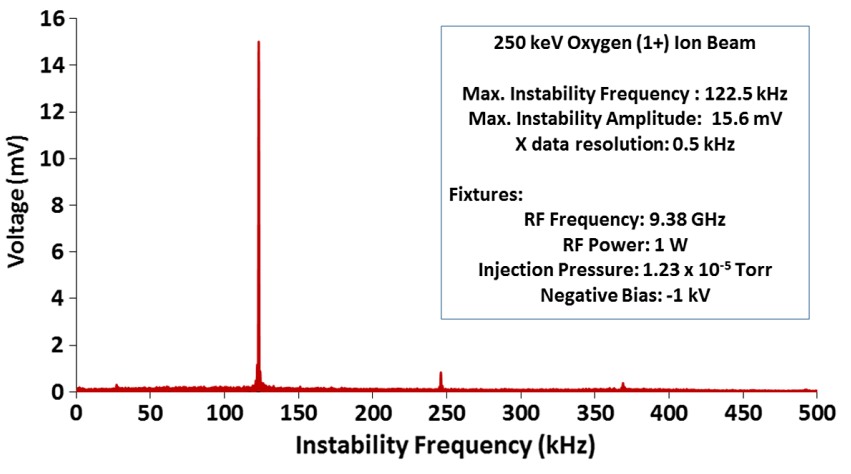

FIG. 3. Maximum plasma instability frequency for the oxygen ion beam under a set of fixtures of ECR source as given in the inset.

amplitude as shown in Fig. 3 under a set of sustainable tuning parameters of ECR source but it may be pushed further if there is more play in the source parameters. The higher harmonics of this frequency are also observed with relatively much weaker amplitudes. The plasma instability results are shown in Fig. 4 under different ECR operating conditions by tuning the four parameters of the source namely injection pressure of the gas $\left(p_{\text {inj }}\right)$, rf power $\left(P_{\mathrm{rf}}\right)$, the negative bias voltage $\left(V_{\text {bias }}\right)$ and the injected radio frequency $\left(f_{\text {rf }}\right)$. These parameter settings are reported in Table I corresponding to instabilities as shown in Fig. 4. The instability amplitudes $\left(\mathbf{V}_{\text {inst }}\right)$ at dominating instability frequency $\left(\mathbf{f}_{\text {inst }}\right)$ are listed in Table II.

A maximum plasma instability amplitude $\approx 45 \mathrm{mV}$ is observed at a frequency of $3.712 \mathrm{kHz}$ as shown in Fig. 4(a). The corresponding transverse beam profiles displaying these beam current oscillations are shown in Fig. 1(b). Thus, the instability amplitude can be more than $10 \%$ of the main dc component of beam current which may affect the source performance. It is optimized further in such a way that a series of its harmonics can be generated in a sustainable manner. The instability frequency of $3.84 \mathrm{kHz}$ along with its higher harmonics of decaying amplitude, is shown in Fig. 4(b). The harmonic generations have been predicted by theoreticians in different types of confined plasmas. Sharma et al. [26] studied a spiraling ion beam propagating through a magnetized inhomogeneous plasma cylinder and predicted higher harmonics of the beam cyclotron frequency in the $\mathrm{kHz}$ range. The instability frequency can also be tuned uniquely and is shown as a single peak at $45.568 \mathrm{kHz}$ in Fig. 4(c). The low-frequency spectrum for ECR plasma with multiple peaks below $400 \mathrm{~Hz}$ is shown in Fig. 4(d). Some oscillations can be seen as harmonics of each other but there are still lots of distinct peaks. The explanation of these lowfrequency peaks is given by a theoretical model which is described in the next section.

\section{TRENDS OF INSTABILITY FREQUENCY}

The effect on plasma instability frequency with rf power is as shown in Fig. 5(a) keeping other parameters 

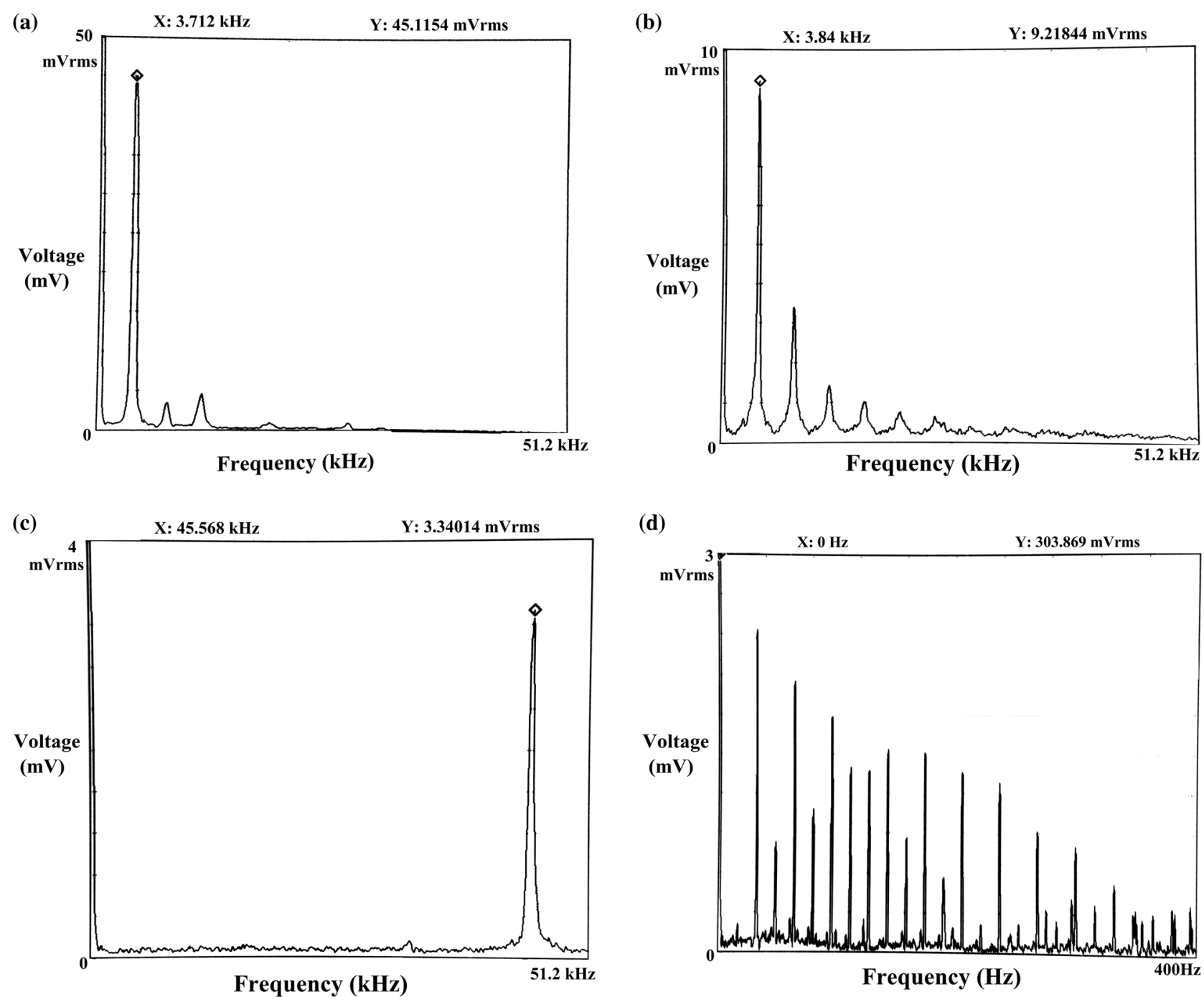

FIG. 4. (a) Generation of maximum amplitude of plasma instability, (b) harmonics generation for plasma instabilities, (c) tunable and unique plasma instability frequency, and (d) low frequency oscillations in ECR ion sources.

$\left(f_{\mathrm{rf}}=11 \mathrm{GHz}, p_{\text {inj }}=10.3 \mu\right.$ Torr, $\left.V_{\text {bias }}=-600 \mathrm{~V}\right)$ of the source as fixed. The instability frequency decreases with increasing rf power which means the generation of a greater number of hot electrons. It is also observed individually with injected radio frequency and gas pressure which shows an oscillating and decreasing behavior, respectively as shown collectively in Fig. 5(b). The similar observation with respect to injected radio frequency is also shown theoretically and experimentally by Gore et al. [11], who

TABLE I. Tuning parameters of ECR ion source for various plasma instabilities generation with respect to Fig. 4.

\begin{tabular}{lcccc}
\hline \hline Parameters & Fig. 4(a) & Fig. 4(b) & Fig. 4(c) & Fig. 4(d) \\
\hline$f_{\text {rf }}(\mathrm{GHz})$ & 9.44 & 9.43 & 9.43 & 9.43 \\
$P_{\text {rf }}(\mathrm{W})$ & 7 & 5 & 3 & 5 \\
$V_{\text {bias }}(\mathrm{V})$ & -99 & -155 & -158 & -133 \\
$p_{\text {inj }}(\mu$ Torr $)$ & 17.6 & 8.59 & 11.9 & 11.9 \\
\hline \hline
\end{tabular}

has shown clearly the oscillating behavior of drift waves with pump frequency of the order of few $\mathrm{MHz}$ corresponding to the vicinity of lower hybrid resonances resulting into drift wave frequency of few $\mathrm{kHz}$. Bhattacharjee et al. [27] studied the fluctuations in ECR plasma in a divergent magnetic field using Langmuir probe diagnostics and showed the same trend of decrease in wave mode frequency with the increase in gas pressure, naming it as falling frequency mode. They have also obtained the strong and nearly coherent low-frequency component along with several harmonic components, naming it rising frequency

TABLE II. Plasma instabilities parameters of various cases with respect to Fig. 4.

\begin{tabular}{lcccl}
\hline \hline Parameters & Fig. 4(a) & Fig. 4(b) & Fig. 4(c) & Fig. 4(d) \\
\hline $\mathbf{V}_{\text {inst }}(\mathrm{mV})$ & 45.12 & 9.22 & 3.34 & 2.44 \\
f $_{\text {inst }}(\mathrm{kHz})$ & 3.712 & 3.84 & 45.568 & 0.03175 \\
\hline \hline
\end{tabular}



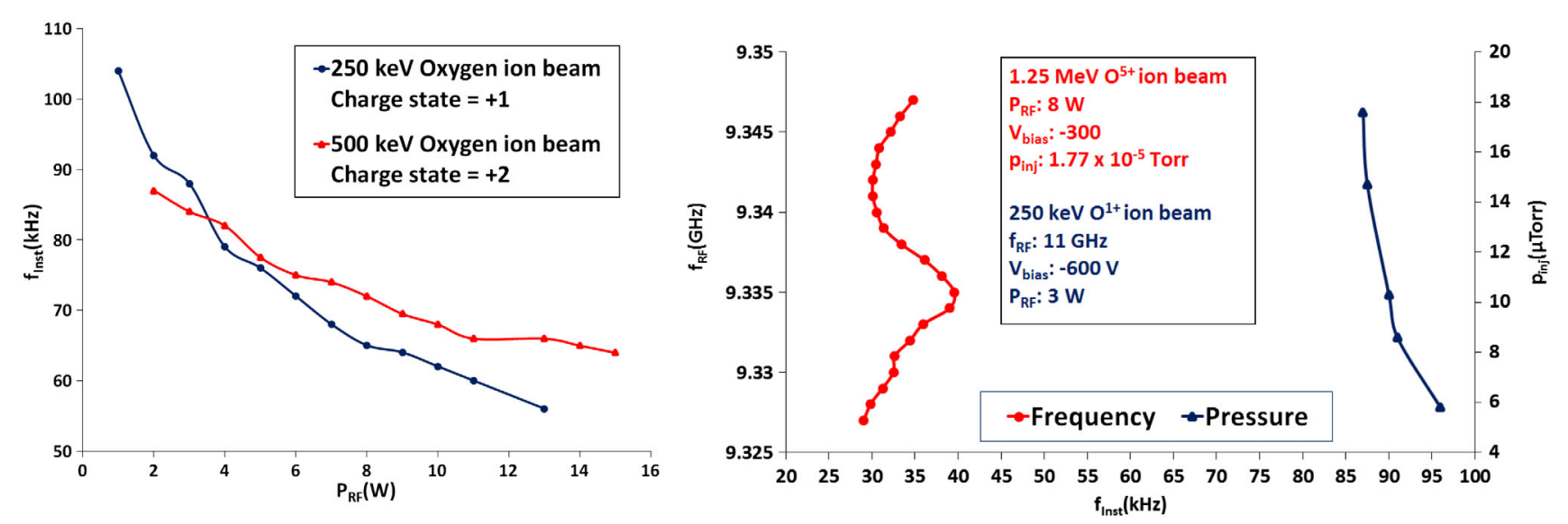

FIG. 5. Effect of (a) rf power and (b) radio frequency and injection pressure of gas on the tunability of plasma instability frequency.

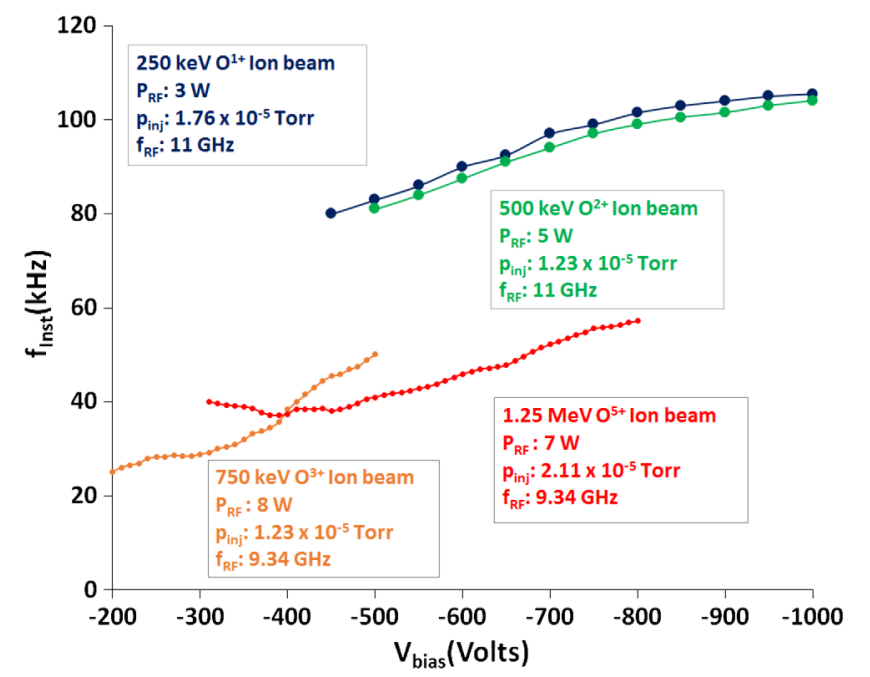

FIG. 6. Effect of bias voltage on the tunability of plasma instability frequency dominating in ECR ion source corresponding to Fig. 4(c) for various charge states of oxygen ion beam.

mode. The drift waves were also named as dissipative modes which seem to be arising at lower gas pressures. Strong low-frequency instabilities were also observed by $\mathrm{Pu}$ et al. [28], who studied the behavior with radio frequency and rf power. They have given a theoretical model which concluded that the instability is also a drift wave type. The instability frequency is found to increase with bias voltage as shown in Fig. 6 for various charge states of the ion beam and can be tuned smoothly over the range of few $\mathrm{kHz}$ to hundreds of $\mathrm{kHz}$ for any charge state of the oxygen ion beam. The negative bias voltage electrode mainly acts as a repeller electrode to repel the electrons back into the plasma.

\section{THEORETICAL JUSTIFICATION}

The drift waves have been found to exist universally in magnetically confined plasmas which exhibit anomalously short confinement times caused by pressure gradient driven instabilities. These have been observed by experimentalists $[7,29,30]$ and calculated by theoreticians $[5,6,31,32]$ for different plasma environments. The plasma instability frequencies of the order of $\mathrm{kHz}$ are in agreement with the theoretical work by Liu et al. [33] but their excitation source is the lower hybrid waves of finite wave number. To match the situation experimentally, the theory of lowfrequency waves in magnetized plasmas due to ion motion is utilized which is given by Goldston et al. [31]. The drift wave $[5,34]$ is a low-frequency plasma instability which is caused due to the free energy provided by a pressure gradient perpendicular to the confining magnetic field in ECR geometry. Let $\omega_{p}$ and $\omega_{c}$ represent the electron plasma and electron cyclotron frequency, respectively. Similarly for ions, $\Omega_{p}$ and $\Omega_{c}$ represent the ion plasma and ion cyclotron frequencies, respectively. In plasmas, there exist many types of waves which propagate with a variety of nonlinear dispersion relations. Such waves of different frequency can travel at different speeds in a dispersive medium. Depending upon the arrangements of electric (E) and magnetic fields (B), different kinds of waves can be generated in a plasma medium. If the waves propagate along the magnetic field $(\mathrm{k} \| \mathrm{B})$ then there are two cases mainly. (i) If $\mathrm{E} \| \mathrm{B}$, they are termed the Langmuir waves. (ii) If $\mathrm{E} \perp \mathrm{B}$, they are known as left and right circularly polarized waves ( $\mathrm{L}$ waves and $\mathrm{R}$ waves).

Using tensor notation, all these waves can be analyzed together. The plasma is considered to have a complex tensor dielectric response. The motion of a plasma fluid is described by a velocity $\left(u_{0}\right)$ with particle of mass $(\mathrm{m})$, charge (q) and density $n_{0}$. Thus, the charge and mass density are $\mathrm{q} n_{0}$ and $\mathrm{m} n_{0}$, respectively. Let there be a pressure perturbation which corresponds to a density perturbation $\left(n_{1}\right)$ via a constant $\gamma$ and plasma temperature (T) as $p_{1}=\gamma \mathrm{T} n_{1}$. Under the action of this perturbation, the equation of state describing the fluid motion is characterized by new velocity $\left(u_{1}\right)$ for such plasmas can be written as follows: 


$$
m n_{0} \frac{\partial u_{1}}{\partial t}=n_{0} q\left(E+u_{1} \times B\right)-\gamma T \nabla n_{1} .
$$

The above continuity equation is linearized and the electrical conductivity tensor $(\underline{\sigma})$ is obtained using Ohm's law. The equation for wave propagation in a nondispersive, isotropic dielectric medium and the dielectric tensor $\underline{\epsilon}$ are given as follows:

$$
\begin{gathered}
\left(\mu_{o} \omega^{2} \underline{\epsilon}-k^{2} \mathbb{X}\right) E=0 \\
\underline{\epsilon}=\epsilon_{0}\left(\mathbb{I}+\frac{i \underline{\sigma}}{\epsilon_{0} \omega}\right) .
\end{gathered}
$$

Here, $\mathbb{X}$ and $\mathbb{I}$ are the tensor matrices, $\mathrm{k}$ is the wave vector and $\mu_{o}$ is the vacuum permeability. The dispersion relation $\left(k_{R}, k_{L}\right.$ versus $\left.\omega\right)$ can be obtained by putting the determinant of the tensor quantity on the left-hand side of Eq. (2) equal to zero and is given as follows:

$$
\begin{aligned}
\frac{c^{2} k_{R}^{2}}{\omega^{2}}=\frac{\Omega_{c}+\Omega_{P}^{2} / \Omega_{c}+\omega}{\Omega_{c}+\omega} & \text { (R-wave) } \\
\frac{c^{2} k_{L}^{2}}{\omega^{2}}=\frac{\Omega_{c}+\Omega_{P}^{2} / \Omega_{c}-\omega}{\Omega_{c}-\omega} & \text { (L-wave) }
\end{aligned}
$$

The shear-Alfvén R-wave has no resonance but the shear-Alfvén L-wave has a resonance at $\omega=\Omega_{c}$. It has been found that both lie in the low-frequency regime for the set of parameters calculated for the Nanogan ECR source. The dispersion relation is plotted in the Fig. 7 where parameters are normalized with respect to electron plasma frequency. It clearly shows that theoretical predictions obtained for analyzing wave propagation in such plasmas are matched to the experimental results of plasma instabilities in the low-frequency regime of the order of $\mathrm{kHz}$ for ECR sources qualitatively.

To account for the low frequency peaks as shown in Fig. 4(d), the Predator-Prey model $[35,36]$ has been used by

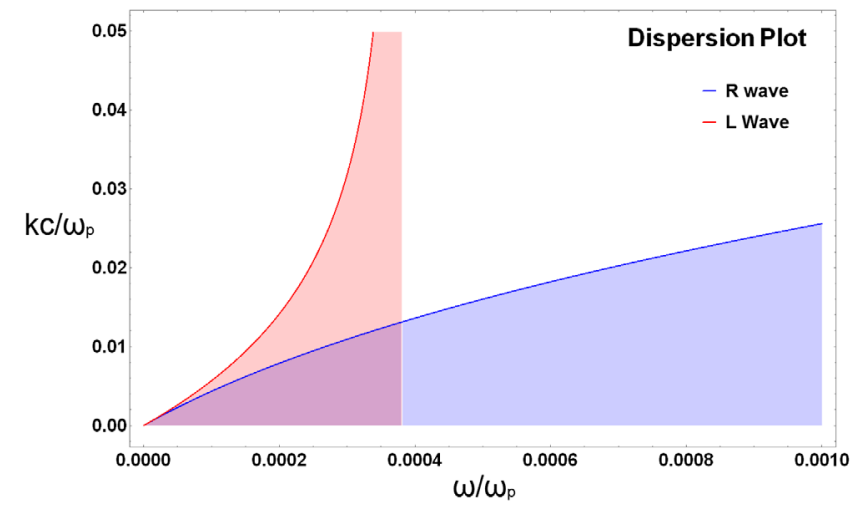

FIG. 7. Dispersion relation for right- and left-hand shearAlfvén waves in the low frequency range. various groups. The conservation equations for ions and neutrals have been solved by taking linear perturbation terms resulting into the final equation of simple harmonic oscillator. Let $D_{\alpha}$ and $D_{n}$ are the ambipolar and neutral diffusion coefficients, respectively, $k_{i}$ is ionization rate coefficients which depends on electron temperature $T_{e}$ and mass, densities, velocities of ions and neutrals denoted as $m_{i}, n_{i}, v_{i}$, and $m_{n}, n_{n}, v_{n}$, respectively. Taking the linear perturbation term as $n_{i}=n_{i, 0}+\varepsilon n_{i}^{\prime}$ and $n_{n}=n_{n, 0}+\varepsilon n_{n}^{\prime}$ and linearizing the conservation equations for ions and neutrals, combining them, it gives a simple harmonic oscillator equation as follows:

$$
\frac{\partial^{2} n_{i}^{\prime}}{\partial t^{2}}+k_{i} n_{i, 0} n_{n, 0} n_{i}^{\prime}=0 .
$$

To zeroth order in $k_{i}, k_{i} n_{i, 0}=D_{\alpha} / L$ and $k_{i} n_{n, 0}=D_{n} / L$. Here $L$ is the extent of plasma zone. The characteristic frequency is given as follows:

$$
\omega=\left(k_{i}^{2} n_{i, 0} n_{n, 0}\right)^{1 / 2}=\frac{\sqrt{D_{\alpha} D_{n}}}{L} .
$$

In case of ambipolar diffusion $D_{\alpha}$, the diffusion mainly occurs from electrons perpendicular to the magnetic field and is given as $D_{\perp e}$. Neutral diffusion coefficients $D_{n}$ are also expressed in terms of parameters for neutrals as follows:

$$
D_{\alpha} \approx D_{\perp e}=\frac{k_{b} T_{e} \nu_{e n}}{m_{i} \omega_{c e}^{2}} ; \quad D_{n}=\frac{k_{b} T_{n}}{m_{n} \nu_{n n}} .
$$

Here $\omega_{c e}$ is the electron cyclotron resonance frequency. $k_{b}$ is the Boltzmann constant. $\nu_{n n}$ and $\nu_{e n}$ are neutralneutral and electron-neutral collisional frequency, respectively. The frequencies as per Eq. (7) are found to be qualitatively within experimental values of the frequencies as shown in Fig. 4(d). The similar results were also observed by the Biswas et al. [37,38], who studied the floating potential measurement in ECR produced plasmas and found the low-frequency oscillations. It may also be explained by nonlinear coupling [39] of wave propagation, neutral and plasma density, microwave power absorption and plasma temperature. Boeuf et al. [40] has also shown theoretically low-frequency oscillations due to periodic depletion of neutral atom density by electron impact ionization in stationary plasma hall thrusters.

\section{PLASMA INSTABILITIES AT HIGHER RF POWER AND CONFINEMENT FIELDS}

For the sake of completeness and usefulness of such plasma studies at higher rf power levels in large volume ECR sources, the instabilities have also been observed experimentally by the same Fourier analysis technique for a high temperature superconducting ECR ion source also known as PKDELIS [41,42] and it has been observed that there is a similar set of plasma instabilities. The source has 


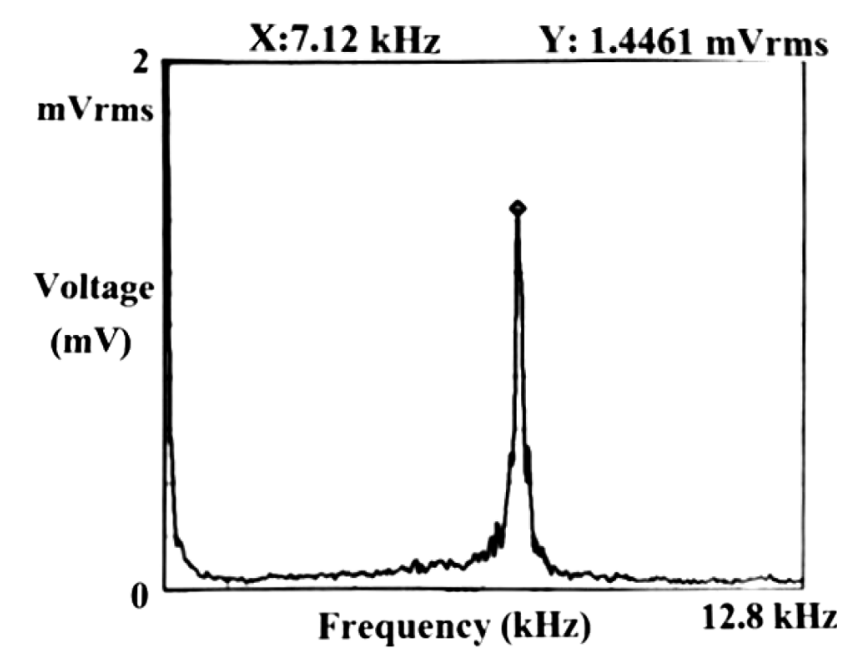

FIG. 8. Plasma instability at $\mathrm{rf}$ power $=250 \mathrm{~W}$, radio frequency $=18 \mathrm{GHz}$, and injection pressure $=1.3 \times 10^{-6}$ torr.

an axial field of $1.8 \mathrm{~T}$ and $1.5 \mathrm{~T}$ at the injection and extraction side, respectively. The operational radio frequency is either $14.5 \mathrm{GHz}$ or $18 \mathrm{GHz}$. The oxygen ion beam at $16.3 \mathrm{kV}$ extraction potential has been analyzed using a 90-degree dipole magnet and measured on a Faraday cup. This particular energy along with $5.03 \mathrm{kV}$ additional acceleration potential is chosen to match $8 \mathrm{keV} / \mathrm{u}$ as injection energy to radio-frequency quadrupole for $\mathrm{O}^{6+}$ ion beam. The instabilities are also generated in this ECR source and one such instability peak is shown in Fig. 8 which is tunable. All the studies reported in Sec. II are at $250 \mathrm{kV}$ electrostatic potential and rf power $\leq 20 \mathrm{~W}$, but here it is at $16.3 \mathrm{kV}$ extraction potential and $\mathrm{rf}$ power $=250 \mathrm{~W}$. Finally, for both low and high volume ECR ion sources, it is observed that plasma instabilities can be generated at any extraction energy of ion beam as well as at any applied rf power in the ECR source sufficient to extract the ion beam and to form plasma, respectively.

\section{CONCLUSION}

The plasma instabilities have been observed clearly as distinct peaks in the Fourier spectrum of the extracted and analyzed beam currents which are tunable with respect to ECR source parameters. The negative bias of inserted plasma electrode and injected rf power were found to be the best parameters to tune the plasma instability frequency within tens of $\mathrm{kHz}$ smoothly. Plasma instabilities, especially the drift waves, are always present at many operating conditions of ECR sources. They exist in both low and high rf power ECR sources and thus make this study useful for characterizing superconducting ECR sources. These instabilities fully govern the trapping of ions for converting into higher charge states. The plasma instabilities are explained by the Predator-Prey model and the R-wave, L-wave concept in different regimes. Further experiments should be carried out in the future to cure them as much as possible by modifying hardware configuration of ECR geometry and optimizing its operational parameters. The reported results of plasma instabilities with respect to tuning parameters of ECR source require more theoretical insights.

\section{ACKNOWLEDGMENTS}

The authors would like to thank the support from the Low Energy Ion Beam Facility (LEIBF) [43] and High Current Injector (HCI) groups of IUAC, New Delhi, for the experiment. They are also thankful to Dr. D. Kanjilal, Director, IUAC Delhi for his useful comments and suggestions. S. K. is thankful to Accelerator User Community (AUC) to issue beam time (Reference No. IUAC/XII.3A/ 62516) for conducting the LEIBF experiment. J.S. is thankful to Department of Science and Technology (DST), New Delhi, for financial assistance vide Grant No. EMR/2016/002699.

[1] R. Geller, Electron Cyclotron Resonance Ion Sources and ECR Plasmas (IOP Publisher, London, 1996).

[2] A. Drentje, Techniques and mechanisms applied in electron cyclotron resonance sources for highly charged ions, Rev. Sci. Instrum. 74, 2631 (2003).

[3] O. Tarvainen, I. Izotov, D. Mansfeld, V. Skalyga, S. Golubev, T. Kalvas, H. Koivisto, J. Komppula, R. Kronholm, J. Laulainen, and V. Toivanen, Beam current oscillations driven by cyclotron instabilities in a minimum-B electron cyclotron resonance ion source plasma, Plasma Sources Sci. Technol. 23, 025020 (2014).

[4] O. Tarvainen, V. Toivanen, J. Komppula, T. Kalvas, and H. Koivisto, Transverse distribution of beam current oscillations of a $14 \mathrm{GHz}$ electron cyclotron resonance ion source, Rev. Sci. Instrum. 85, 02 A909 (2014).

[5] W. Horton, Drift waves and transport, Rev. Mod. Phys. 71, 735 (1999).

[6] F. C. Francis et al., Introduction to Plasma Physics and Controlled Fusion, Plasma Physics, Vol. 1 (Springer Science \& Business Media, USA, 1984).

[7] H. W. Hendel, B. Coppi, F. Perkins, and P. A. Politzer, Collisional Effects in Plasmas-Drift-Wave Experiments and Interpretation, Phys. Rev. Lett. 18, 439 (1967).

[8] T. K. Chu, B. Coppi, H. W. Hendel, and F. W. Perkins, Drift instabilities in a uniformly rotating plasma cylinder, Phys. Fluids 12, 203 (1969).

[9] A. Semet, A. Mase, W. A. Peebles, N. C. Luhmann, and S. Zweben, Study of Low-Frequency Microturbulence in the Microtor Tokamak by Far-Infrared Laser Scattering, Phys. Rev. Lett. 45, 445 (1980).

[10] J. Weiland and H. Nordman, Drift wave model for inward energy transport in tokamak plasmas, Phys. Fluids B 5, 1669 (1993).

[11] R. Gore, J. Grun, and H. Lashinsky, Stabilization of Drift Waves by Lower-Hybrid Fields, Phys. Rev. Lett. 40, 1140 (1978).

[12] G. Praburam, H. Honda, and M. Sugawa, Suppression of drift waves in an ion beam-plasma system, J. Phys. Soc. Jpn. 62, 4262 (1993). 
[13] Y. B. Fainberg and V. Shapiro, Drift instabilities of a plasma situated in a high-frequency electric field, Sov. Phys. JETP 25, 189 (1967).

[14] M. Okamoto, T. Amano, and K. Kitao, Effects of a highfrequency electric field on micro-instabilities driven by density gradient, J. Phys. Soc. Jpn. 29, 1041 (1970).

[15] R. Schrittwieser, N. Rynn, R. Koslover, and R. Karim, Electrostatic ion-cyclotron instability driven by a slow electron drift, Plasma Phys. Controlled Fusion 26, 1597 (1984).

[16] W. E. Drummond and M. N. Rosenbluth, Anomalous diffusion arising from microinstabilities in a plasma, Phys. Fluids 5, 1507 (1962).

[17] J. Sharma and S. C. Sharma, Excitation of electrostatic ioncyclotron waves by an ion beam in a two-ion component plasma, Phys. Plasmas 17, 123701 (2010).

[18] D. M. Suszcynsky, N. D'Angelo, and R. L. Merlino, An experimental study of electrostatic ion cyclotron waves in a two-ion component plasma, J. Geophys. Res. 94, 8966 (1989).

[19] J. Sharma, S. C. Sharma, V. K. Jain, and A. Gahlot, Excitation of lower hybrid waves by a gyrating ion beam in a negative ion plasma, Phys. Plasmas 20, 033706 (2013).

[20] D. Kanjilal, T. Madhu, G. Rodrigues, U. Rao, C. Safvan, and A. Roy, Development of a low energy ion beam facility at NSC, Indian J. Pure Appl. Phys. 39, 25 (2001).

[21] P. Sortais, M. Bisch, M. Bourgarel, P. Bricault, P. Lehérissier, R. Leroy, J. Pacquet, and J. Rataud, Latest developments on multicharged E. C. R. ion sources at GANIL, in EPAC 92-Third European Particle Accelerator Conference, Berlin, Germany (1992), pp. 990-992.

[22] P. Kumar, G. Rodrigues, U. K. Rao, C. P. Safvan, D. Kanjilal, and A. Roy, ECR ion source based low energy ion beam facility, Pramana 59, 805 (2002).

[23] S. Kumar and A. Mandal, Low energy ion beam dynamics of NANOGAN ECR ion source, Nucl. Instrum. Methods Phys. Res., Sect. A 814, 73 (2016).

[24] R. Baskaran, T. S. Selvakumaran, G. Rodrigues, D. Kanjilal, and A. Roy, Measurements and analysis of bremsstrahlung $\mathrm{X}$-ray spectrum obtained in NANOGAN electron cyclotron resonance ion source, Rev. Sci. Instrum. 79, 02 A324 (2008).

[25] G. Rodrigues, K. Mal, N. Kumar, R. Baskaran, P. S. Lakshmy, Y. Mathur, P. Kumar, D. Kanjilal, and A. Roy, Effect of frequency tuning on bremsstrahlung spectra, beam intensity, and shape in the $10 \mathrm{GHz}$ NANOGAN electron cyclotron resonance ion source, Rev. Sci. Instrum. 85, 02 A944 (2014).

[26] J. Sharma, S. C. Sharma, V. Jain, and A. Gahlot, Higher harmonics generation by a spiraling ion beam in collisionless magnetized plasma, J. Plasma Phys. 79, 577 (2013).

[27] S. Bhattacharjee, A. Fredriksen, and S. Chandra, Fluctuations in electron cyclotron resonance plasma in a divergent magnetic field, Phys. Plasmas 23, 022109 (2016).

[28] Y. K. Pu and W. Halverson, Plasma instability in ECR heated ion sources, in 1990 IEEE international conference on plasma science-Conference Record-Abstracts (Spire Corp., Bedford, MA, 1990), p. 160.

[29] H. W. Hendel, T. Chu, and P. Politzer, Collisional Drift Waves-Identification, Stabilization, and Enhanced Plasma Transport, Phys. Fluids 11, 2426 (1968).
[30] J. Anderson, T. Rafiq, M. Nadeem, and M. Persson, A comparison of drift wave stability in stellarator and tokamak geometry, Phys. Plasmas 9, 1629 (2002).

[31] R. J. Goldston and P. H. Rutherford, Introduction to Plasma Physics (CRC Press, Boca Raton, FL, 1995).

[32] A. Borgohain and P. Deka, Electrostatic instability in magnetically confined inhomogeneous plasma driven by nonlinear force, Phys. Lett. A 378, 790 (2014).

[33] C. S. Liu and V. K. Tripathi, Stabilization of drift waves by lower hybrid waves of finite wavenumber, Phys. Fluids 23, 345 (1980).

[34] K. Kamataki, Y. Nagashima, S. Shinohara, Y. Kawai, M. Yagi, K. Itoh, and S.-I. Itoh, Coexistence of collisional drift and flute wave instabilities in bounded linear ECR plasma, J. Phys. Soc. Jpn. 76, 054501 (2007).

[35] J. Fife, M. Martinez-Sanchez, and J. Szabo, A numerical study of low-frequency discharge oscillations in Hall thrusters, 33rd Joint Propulsion Conference and Exhibit, Joint Propulsion Conferences, Seattle,WA (American Institute of Aeronautics and Astronautics, 1997), https://doi .org/10.2514/6.1997-3052.

[36] E. Y. Choueiri, Plasma oscillations in Hall thrusters, Phys. Plasmas 8, 1411 (2001).

[37] S. Biswas, R. Pal, and A. Iyengar, Very low frequency oscillations in ECR produced plasmas of the MaPLE device, in General Assembly and Scientific Symposium, 2011 XXXth URSI (IEEE, New York, 2011), pp. 1-4.

[38] S. Biswas, D. Basu, R. Pal, and N. Chakrabarti, Selective Excitation of Low Frequency Drift Waves by Density Modulation and Parametric Excitation of Higher Frequency Mode, Phys. Rev. Lett. 111, 115004 (2013).

[39] E. S. Aydil, J. A. Gregus, and R. A. Gottscho, Multiple steady states in electron cyclotron resonance plasma reactors, J. Vac. Sci. Technol. A 11, 2883 (1993).

[40] J. P. Boeuf and L. Garrigues, Low frequency oscillations in a stationary plasma thruster, J. Appl. Phys. 84, 3541 (1998).

[41] D. Kanjilal, G. Rodrigues, P. Kumar, A. Mandal, A. Roy, C. Bieth, S. Kantas, and P. Sortais, Performance of first high temperature superconducting ECRIS, Rev. Sci. Instrum. 77, 03A317 (2006).

[42] G. Rodrigues, P. S. Lakshmy, S. Kumar, A. Mandal, D. Kanjilal, R. Baskaran, and A. Roy, Studies of emittance of multiply charged ions extracted from high temperature superconducting electron cyclotron resonance ion source, PKDELIS, Rev. Sci. Instrum. 81, $02 B 713$ (2010).

[43] See Supplemental Material at http://link.aps.org/ supplemental/10.1103/PhysRevAccelBeams.21.093402, for Real Picture of Nanogan ECR ion source, its extraction system, high voltage Deck and the accelerating section, the background spectra of noise for Fourier analysis is less than $10 \mu \mathrm{V}$.

Correction: The numerical entries in the first row of Table II have been adjusted to correlate with values in Fig. 4. A typographical error in the inline equation appearing in the first sentence after Eq. (5) has been corrected. 